\title{
A Data Processing Middleware Based on SOA for the Internet of Things
}

\author{
Feng Wang, Liang Hu, Jin Zhou, and Kuo Zhao \\ College of Computer Science and Technology, Jilin University, Changchun 130012, China \\ Correspondence should be addressed to Kuo Zhao; zhaokuo@jlu.edu.cn
}

Received 17 September 2014; Revised 11 January 2015; Accepted 27 January 2015

Academic Editor: Fei Yu

Copyright (C) Feng Wang et al. This is an open access article distributed under the Creative Commons Attribution License, which permits unrestricted use, distribution, and reproduction in any medium, provided the original work is properly cited.

\begin{abstract}
The Internet of Things (IoT) emphasizes on connecting every object around us by leveraging a variety of wireless communication technologies. Heterogeneous data fusion is widely considered to be a promising and urgent challenge in the data processing of the IoT. In this study, we first discuss the development of the concept of the IoT and give a detailed description of the architecture of the IoT. And then we design a middleware platform based on service-oriented architecture (SOA) for integration of multisource heterogeneous information. New research angle regarding flexible heterogeneous information fusion architecture for the IoT is the theme of this paper. Experiments using environmental monitoring sensor data derived from indoor environment are performed for system validation. Through the theoretical analysis and experimental verification, the data processing middleware architecture represents better adaptation to multisensor and multistream application scenarios in the IoT, which improves heterogeneous data utilization value. The data processing middleware based on SOA for the IoT establishes a solid foundation of integration and interaction for diverse networks data among heterogeneous systems in the future, which simplifies the complexity of integration process and improves reusability of components in the system.
\end{abstract}

\section{Introduction}

The concept of the Internet of Things (IoT) was firstly derived by the Automatic Identification (Auto-ID) Labs in the Massachusetts Institute of Technology (MIT) in 1999. The Auto-ID Labs simultaneously propose [1] radio frequency identification (RFID) systems that connect devices and transmit information via radio frequency to the Internet in order to achieve intelligent identification and management. To formalize the concept of the "Internet of Things," the International Telecommunication Union (ITU) released the report of "ITU Internet reports 2005: the Internet of Things [2]" in the World Summit on Information Society (WSIS) held in Tunis in 2005, in which the IoT characteristics, related technical challenges, and future market opportunities were introduced.

ITU pointed out in the report [2], "We are standing at the edge of new times of communication, information and communication technology (ICT) to achieve the objectives have been developed to meet the communication between people, and things, between things connection. The coming era of ubiquitous Internet of Things make us a new dimension of communication in the world of information and communication technology (shown in Figure 1), any time, any place, connected to anyone, expansion to connect things connected to the Internet of Things."

With the rapid development of information and communication technology, just a onefold technology cannot satisfy the complex context-aware application requirements, in which resource information has been subject to outside interference, and people want to be able to obtain realtime and real-world information such as diverse sensory data acquisitions and human-computer interaction data acquisitions and ultimately achieve efficient data acquisitions between people and things, people, and things and things.

The IoT applications integrate with IntelliSense recognition technologies, pervasive computing, and ubiquitous networks, which are called the third wave of the information technology revolution following the development of the information industry in the computer and the Internet. The IoT is an important part of the new generation of information 


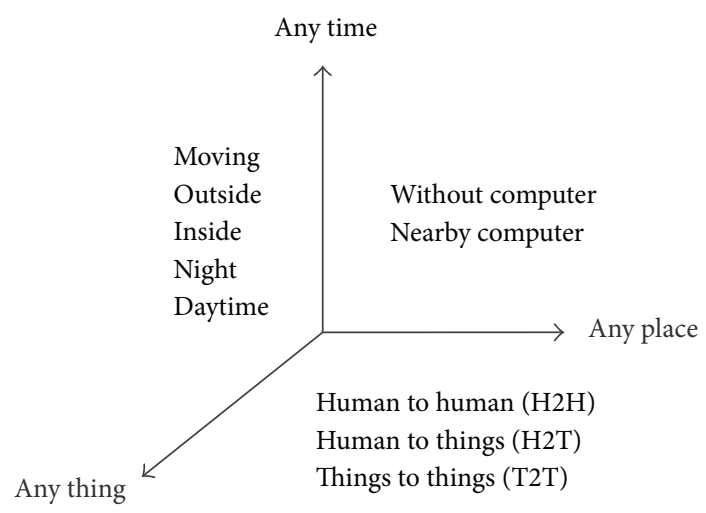

FIgUre 1: Connections in the IoT.

technology. The foundation of the IoT is still the Internet and it is based on the extension and expansion of Internet. The IoT extends its client to anything and any person by the exchange of information and communication though Internet. The IoT connects everything to the Internet.

The IoT incorporates RFID, wireless sensor networks, and ubiquitous terminal equipment as the perception foundation, with a variety of wired or wireless communication and integration of the Internet to achieve the perception data transferred and shared. By leveraging cloud computing and highperformance computing technology for real-time information process, management, and organization, we ultimately offer the upper application a variety of feedback decisionmaking processes for closed-loop control of the things.

Consequently, the growing popularity of the IoT will inevitably lead to a new wave of development of various industries, such as smart home, intelligent monitoring, smart grid, and other new concepts of things technology. So far, the IoT has been launched as a variety of demonstration applications in different domains (shown in Figure 1), such as intelligent industry [3], intelligent agriculture [4], intelligent logistics [5], intelligent transportation [6], smart grid [7], environmental protection [8], security protection [9], intelligent medical care [10,11], and smart home [12].

The rest of the paper is organized as follows. In Section 2, the IoT concepts are reviewed. The description of the architecture of the IoT is detailed in Section 3. In Section 4, we propose a middleware framework based on SOA for IoT. We conclude the paper and point out future work in Section 5.

\section{Related Work}

The IoT will be a promising facility of future network which has self-configuration ability in global dynamic network based on standard and interoperable communication protocols. In the network, all real and virtual items have specific identification and physical sensory data in order to achieve the goal of information sharing through seamless connection of intelligent interface [13, 14]. These intelligent interfaces connect and communicate with users, society, and environment context on the basis of the agreed protocols. It is an extension and expansion of the network based on the
Internet to achieve intelligent identifying, locating, tracking, monitoring, and managing.

From an alternative perspective beyond the initial concept of the IoT and the definitions of it as abovementioned, the IoT is a network connecting things to things for achieving intelligent identification and management of the items in a broad sense; it can be seen as a fusion of the information space and physical space. Through that way, everything is digitized and networked, which results in realizing an efficient information interactive mode between items, items and people, and people and environment. After that, various diversities of information are merged into social networks and integrated into human society in a higher realm. For realization of information fusion in the IoT, the middleware technology is suitable to be adopted as a concrete solution.

Middleware as computer software provides connection of different software components and applications. It consists of a set of enabling services that allow multiple processes running on one or more machines to interact across a network. Atzori et al. [13] summarized the relationship as three visions of the IoT, that is, things-oriented visions, semantic-oriented visions, and Internet-oriented visions. According to the three characteristics, middleware in the IoT shall be able to address things issues and Internet issues, deal with the semantics gap, such as interoperability across heterogeneous devices, context awareness, and device discovery, manage resources constrained embedded devices and scalability, manage large data volumes and privacy, and cope with semantic data and so forth.

Several studies have been published that have explored ways to design middleware for the IoT. In [15], Römer et al. summarized the functions and the nature of the middleware for wireless sensor network. In [16], Wang et al. have reviewed middleware for WSN and a detailed analysis of the approaches and techniques offered by the middleware to meet the requirements of the WSN has been presented. It also discusses generic components of the middleware and reference model of WSN based middleware. In [17], middleware has been surveyed from adaptability perspective in which Sadjadi and McKinley presents taxonomy for adaptive middleware and their application domains and provides details for one of each middleware category. The context-awareness middleware also has been studied. The survey in [18] is based on the architectural aspects and provides taxonomy of the features of the generic context-aware middleware. A survey reported in [19] evaluates several context-aware architectures based on some relevant criteria from ubiquitous or pervasive computing perspective. In [20], Bandyopadhyay et al. provides a survey of middleware system for the IoT.

\section{The Architecture of the IoT}

Heterogeneous information sources are the most important characters of the IoT. In order to achieve interconnection, intercommunication, and interoperability between heterogeneous information, the future architecture of the IoT needs to be open, layered, and scalable [21]. The IoT architecture is generally divided into four layers that are perception 


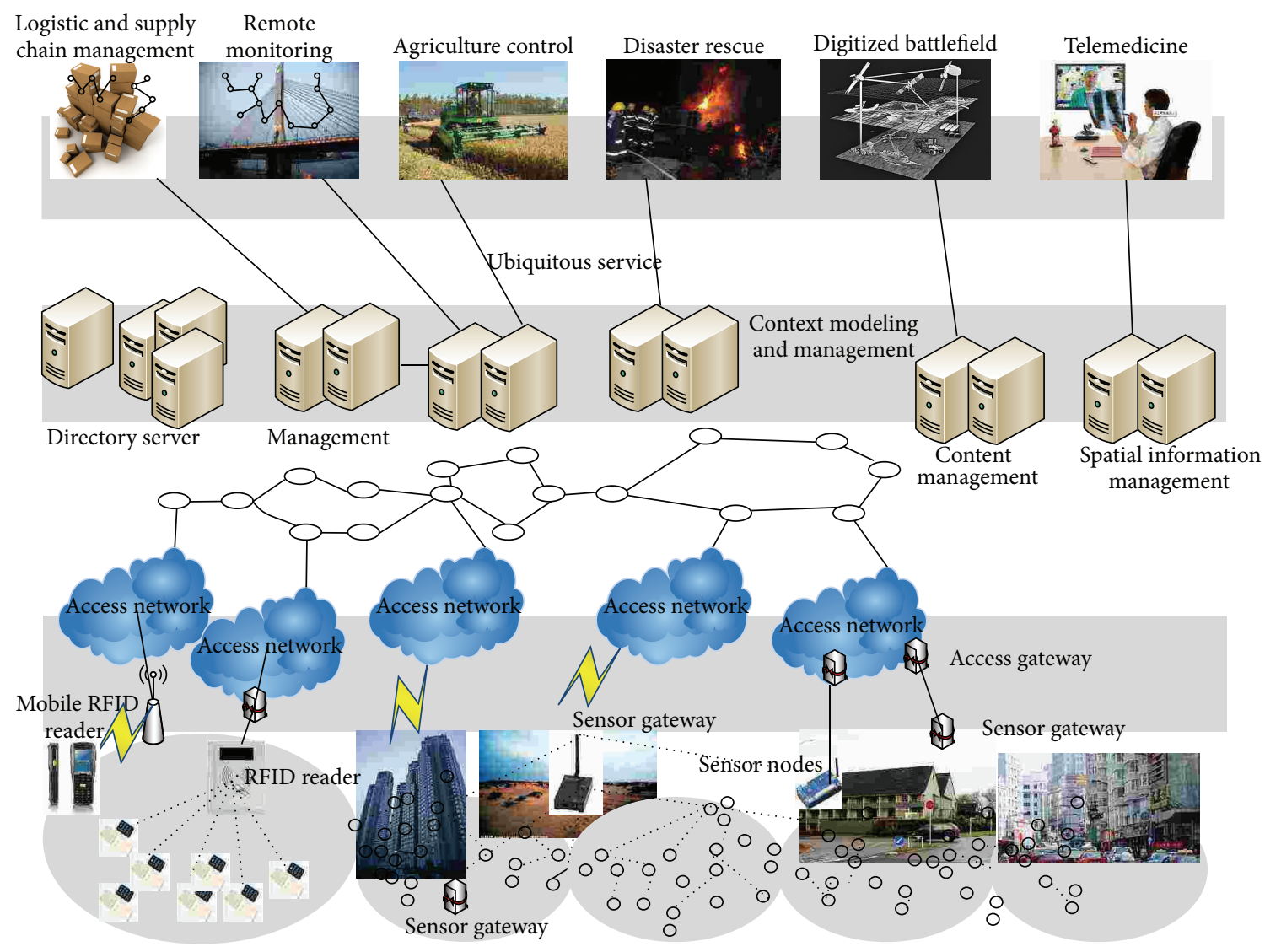

FIGURE 2: Layered framework in the IoT.

layer, network layer, middleware layer, and application layer (shown in Figure 2).

A major part of perception layer is wireless sensor nodes. A generic sensor node aims to take measurements of physical environment [22]. It may be equipped with a variety of devices which can measure various physical attributes such as light, temperature, humidity, barometric pressure, acceleration, acoustics, magnetic field, and carbon dioxide concentration. In addition to the sensors, perception layer also consists of a large amount of information generated equipment, including RFID and positioning systems, and a variety of smart devices, such as smart phones, PDAs, multimedia players, netbooks, and laptops. It can be seen that the diversity of generated information is an emerging and important feature of the IoT.

IPv6 addresses the major defect of the limit on the number of terminal pieces of equipment to access Internet. The main idea of network layer is leveraging the existing Internet as the main dissemination of information by virtue of a variety of wireless accesses. Every wireless access method has its own characteristics and application scenarios. Wi-Fi and other wireless broadband technologies possess broader coverage, faster transmission, reliable high-speed, and lower cost and circumvent the obstacles. The low-speed wireless networks, such as ZigBee, Bluetooth, and infrared low-speed network protocols, are adapted to resource constrained node, which has the characteristics of the low communication radius, low computing power, and low energy consumption. Mobile communication network will become an effective platform for "a comprehensive, anytime, anywhere."

Middleware layer tackles the information heterogeneity issues by intelligent interfaces. The functional solutions of middleware layer mainly consist of data storage (database and mass storage technology), heterogeneous data retrieval (search engine), data mining, data security, and privacy protection.

In application layer, traditional Internet has gone through data-centric to people-centric conversion; typical online applications include file transfer, e-mail, the World Wide Web, e-commerce, online gaming, and social networking. In the application of IoT, things or physical world are considered as the center, typical IoT applications covering item tracking, context-aware, intelligent logistics, intelligent transportation, smart grid, and so forth. The IoT application is currently in a period of rapid growth.

\section{The Implementation of Middleware Based on SOA for IoT}

The IoT research mainly pays more attention to network layer recently, such as the IoT network coding, identification and anticollision technology. However, data processing 
infrastructure continues to be overwhelmed by the mass of heterogeneous information from the number of terminals in the IoT. The flexible architecture that is based on SOA for heterogeneous information fusion in the IoT offers the opportunity to employ mitigation measures. It is critical for the ultimate success of the IoT application for better utilization of the integration of a wide range of services from multiple sources and provides more personalized service to businesses or individuals [9].

4.1. Service-Oriented Application Architecture Description. SOA (service-oriented architecture) is a component model and links different functional units (called services) of the application through well-defined interfaces and contracts between these services. Interface is defined by a neutral manner and it should be independent of implementation services, hardware platforms, operating systems, and programming languages. This allows the service to be built in a variety of such systems to interact in a uniform and general way [23]. The service is the basis of the SOA; thereby, they can be applied directly and effectively depending on system and interaction of software agents.

Typically, business operations running in an SOA comprise a number of different components, which are often in an event-driven or asynchronous fashion that reflects the underlying business process needs [24]. In the context of the IoT, original and emerging resources are in the form of services and are opening on Internet. Consequently, the study of SOAbased fusion application technology is of great value [25].

SOA architecture consists of five main parts, depicted as below:

(1) Consumer: acquires the information from producers' entities that provide services, such as mobile terminals and web clients.

(2) Application: provides application interfaces or different degrees of loosely coupled services, such as mobile applications, web applications, and rich client.

(3) Service: the implementation of the entities involved in a specific task, such as data center and enterprise information center.

(4) Service Support: SOA specific application background support functions, such as security, management, and semantic analysis.

(5) Producer: an entity to provide specific services or functions.

4.2. The IoT Middleware Design. Inspired by the characteristics of data in the IoT, the design of middleware with the service-oriented architecture was employed in this paper, and integration services, compatible with various types of data and the agreement has been divided. Consequently, this paper presents the basic framework of SOA-based IoT applications as shown in Figure 3. In Figure 3, the three-layer structure of the original SOA is broken down into a five-layer system. Service providers (producers) use of various types of environmental sensing technology. Data processing platform

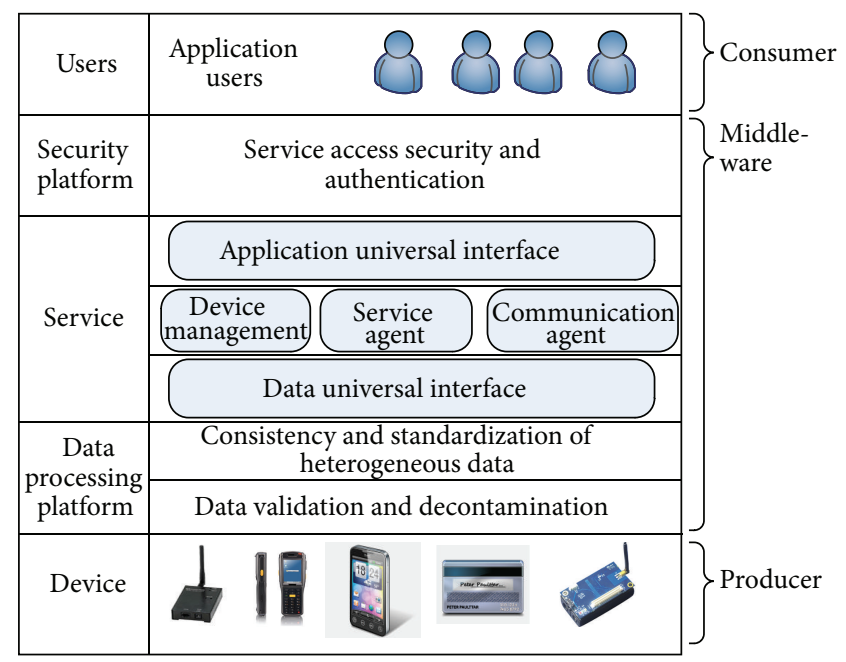

FIGURE 3: The IoT middleware architecture based on SOA.

is responsible for data processing, data filtering, and data integrity. It provides XML scheme for data unification and metadata consistency and standardization of heterogeneous data processing.

Security platform is a security barrier between the service platform and data platform, which is responsible for the safety of the equipment and data. Service layer aims at providing a range of generic interfaces and agency services which are responsible for data parsing in order to coordinate different data formats and are also advantageous for distributed deployment of a variety of databases. The purpose of universal interface is to achieve compatible communication protocols, which are used by different types of users, to perform unified data exchanging with the upper consumers.

The key part of the service layer is to form a bridge between the data processing and the upper application. The service layer also faces different problems that are encountered in the IoT application such as network connection, resource-constrained nodes, and different application platform. Because the underlying device is extremely rich in the IoT, the SOA system to provide network services needs to consider the problem of transmission delay and resource scheduling and network services need to provide a variety of routing or delay tolerant network technology to deal with. SOA systems also need a balanced scheduling algorithm and balanced network resources. Different application platforms require more generic SOA system design patterns; we will first consider the standard between different devices and the upper users between different access platforms.

As it can be seen from Figure 3, the basic framework of SOA application is on the basis of the data stream generated by the perceive network from the physical world, which is with the basic physical properties of the world from the underlying environmental sensing. In SOA architecture, these vast amounts of real context-aware data form the basis of the entire application.

Since heterogeneous data processing is inevitably linked to the IoT middleware architecture based on SOA, a concrete 


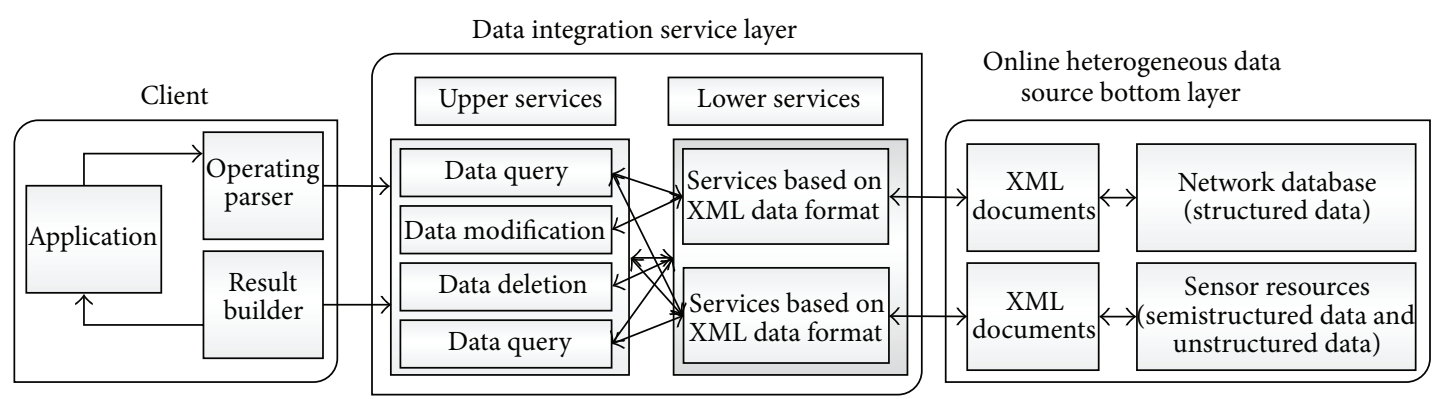

FIgURE 4: The IoT data integration middleware based on SOA.

solution is proposed for the metadata integration of heterogeneous data which is as shown in Figure 4. This architecture is divided into three basic processes, which from top to bottom are the client application layer, the data integration layer, and the IoT heterogeneous data sources, as shown in Figure 4.

The client applications include users unified access interface for data manipulation, which can be a specific application or a web browser.

Data integration service layer [26] is the core of the architecture and also the key to heterogeneous data integration. In order to increase the intelligence and scalability of architecture and alleviate the burden on users, we design a structure that contains the upper and lower levels of service. Metadata format vary greatly since which are grounded in heterogeneous sensor sources [27]. To circumvent this obstacle, we express various types of data into XML format and set up rules to make operations on the metadata. Consequently, we first converted the heterogeneous data in a unified XML format, on this basis, and then created the underlying data integration services layer. The upper layer services built on top of the lower layer services and the underlying services are developed in an XML document based on the underlying heterogeneous data sources.

Services of lower levels achieve four data access functions: add, modify, query, and delete and the upper service function extracts the same functionality from the underlying service to the same service according to the data then forms the integrated data service function. Application layer call corresponding upper services according to the operational requirements, and then underlying data manipulation is specified by the upper service based on data parameters from the client calls; thus, when the underlying heterogeneous data source changes, we simply update the underlying service and map to the upper applications rather than make any changes to upper layers. Data integration implementation process is completely transparent to the user, which is compatible and interoperable in different systems.

4.3. Evaluation. In order to test the IoT middleware architecture based on SOA, we set up an indoor temperature monitoring system in practical environment. We deploy 30 sensor nodes in three rooms, to monitor the indoor temperatures. After collection of the sensor data, the data is delivered by a wireless multihop network from sensor nodes to a base station which is connected to a cluster.
In experiments, clusters include 4 common PCs that are 2-core $2.8 \mathrm{GHz}$ desktop computer with $2 \mathrm{~GB}$ RAM and Ubuntu operating system for the runtime environment. Although they are generic computers, they satisfy the requirements.

In the paper, the sensor nodes are IRIS nodes. IRIS nodes are produced by Crossbow Technology Inc. These sensors are based on the Atmegal1281 microprocessing chip and a RF230 RF chip which are working at $2.4 \mathrm{GHz}$ and supporting the IEEE 8.2.15.4 communication protocol. The nodes have three times radio range and twice the program memory of MICA Motes and outdoor line-of-sight tests have a range as great as 500 meters between nodes without amplification. The IRIS not only has a longer transmission distance but also has ultralow power consumption and a longer battery life.

In the test, equipment used is shown in Figure 5 and the portal of the IoT system for environment monitoring is shown in Figure 6. Some example of the collected sensory data is as shown in Table 1. The monitoring system continues evaluating for three months and collects about five million of the sensor data items. During the test period, we have supplemented and changed several types of the sensors without interrupting the system. When we add the new equipment, some new profiles will be added to the middleware framework while the system is still running.

We can draw some conclusions from the result of the experiment.

First, the SOA-based data processing middleware architecture represents better adaptation to the IoT multisensor and multistream application scenarios, which improve the heterogeneous data reusability and utilization value.

Second, the experiment result demonstrates the decoupling power of middleware which makes it easier to establish a unified heterogeneous information processing platform for a diversity of applications in the IoT.

Third, the distributed deployment of middleware brings better performance optimization and achieves better load balancing in the cluster.

\section{Conclusion}

This paper discusses the development of the concept of the IoT and gives a detailed description of the architecture of the IoT. Based on characteristics of the architecture and challenges of information fusion in the IoT, the paper designs a middleware platform based on SOA architecture for the 
TABLE 1: Some examples of the collected sensory data.

\begin{tabular}{lccccccccccc}
\hline Node ID & Parent & Board_ID & Voltage & Humid & hum temp & pr temp & Press & accel_ $x$ & accel_ $y$ & accel_z & Time \\
\hline 3 & 4 & 133 & 2988 & 27 & 23 & 22.13008 & 939.5078 & 4062 & 125 & 994 & $33: 05.8$ \\
9 & 0 & 133 & 2996 & 27 & 24 & 22.42129 & 940.9485 & 19 & 140 & 1010 & $32: 57.8$ \\
6 & 9 & 133 & 2389 & -4 & -39 & 17.67822 & 931.6279 & 4086 & 64 & 1026 & $27: 08.9$ \\
8 & 0 & 133 & 2345 & -4 & -39 & 19.73633 & 943.171 & 57 & 84 & 3076 & $41: 28.3$ \\
10 & 0 & 133 & 1950 & -4 & -39 & 11.34609 & 938.7853 & 58 & 4094 & 3142 & $16: 08.9$ \\
2 & 0 & 133 & 1911 & 1 & -39 & 19.94141 & 937.0197 & 209 & 42 & 3119 & $32: 47.7$ \\
5 & 1 & 133 & 1917 & 15 & -39 & 16.5043 & 942.0411 & 33 & 55 & 3111 & $07: 09.5$ \\
1 & 9 & 133 & 2728 & 53 & 20 & 18.78281 & 942.9518 & 3847 & 104 & 1018 & $29: 46.8$ \\
7 & 9 & 133 & 2981 & 28 & 23 & 21.90244 & 940.4976 & 4071 & 83 & 994 & $30: 14.3$ \\
4 & 0 & 133 & 3017 & 26 & 24 & 21.84063 & 941.601 & 4088 & 106 & 1017 & $33: 07.9$ \\
0 & 9 & 133 & 3025 & 35 & 22 & 20.2375 & 933.5154 & 4079 & 136 & 1010 & $10: 37.8$ \\
\hline
\end{tabular}
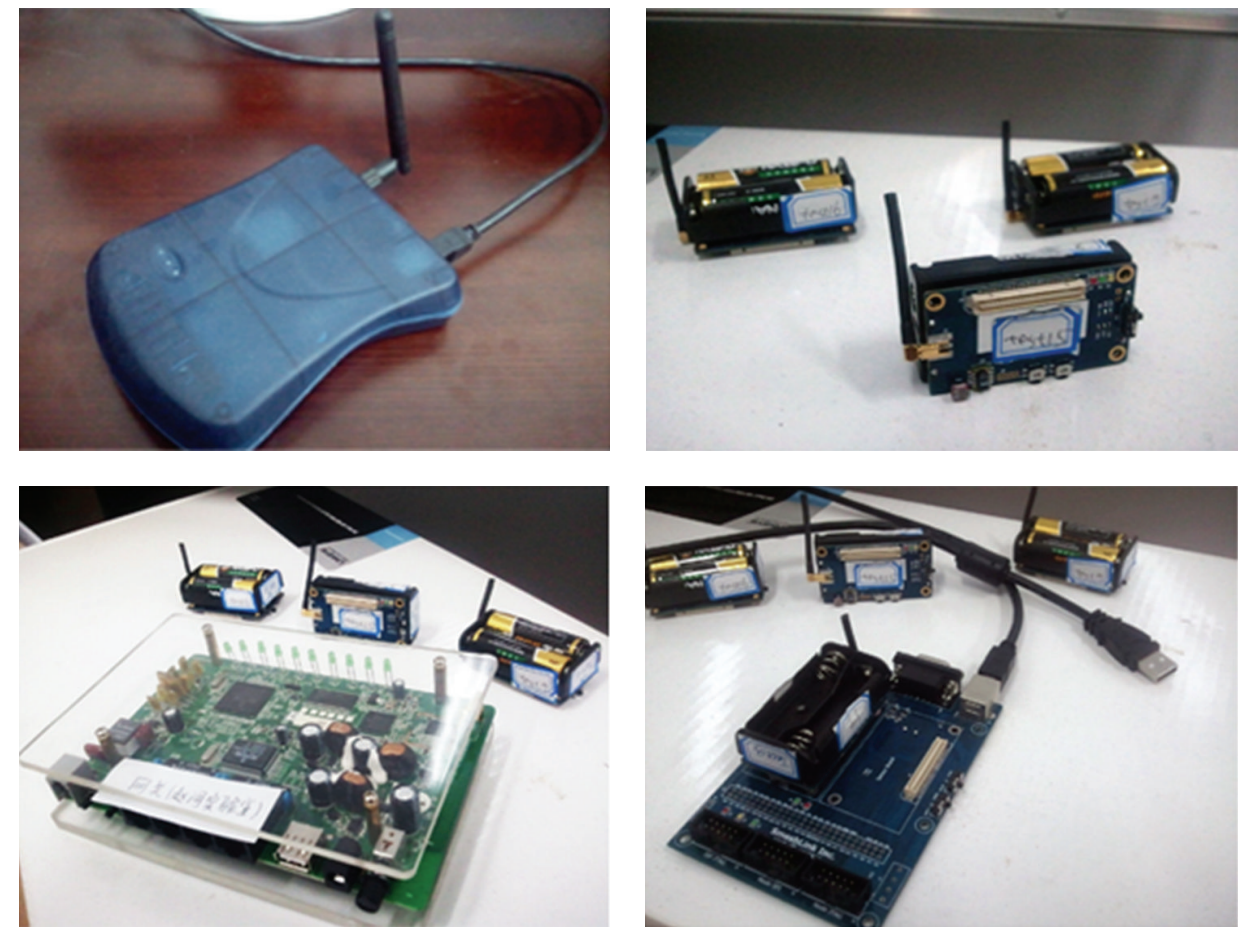

FIgURE 5: The equipment used in the demo.

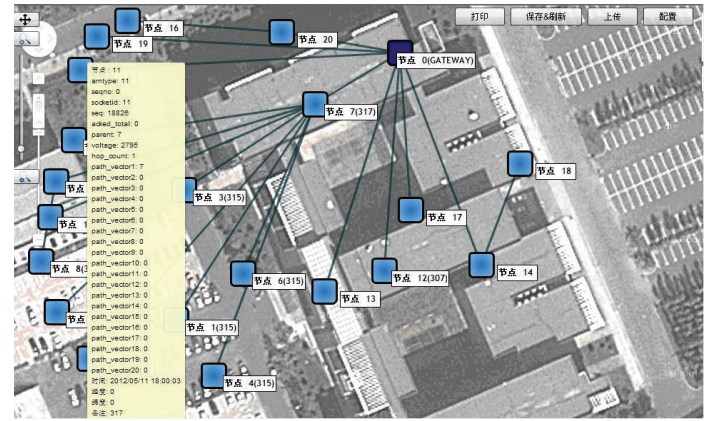

FIgURE 6: The portal of the IoT system for environment monitoring. integration of multisource heterogeneous information. After that, we use the SOA data processing middleware to build an environmental monitoring system for validation verifying. Through theoretical analysis and experimental verification, the SOA pattern-based processing middleware architecture design is better adapted to the IoT multisensor and multistream application scenarios, which improve the sensing data utilization value. The SOA data processing middleware has laid a solid foundation for data integration and interaction between different networking systems, simplifying the complexity of the system integration process and improving the reuse of components in the future. In order to achieve better 
interaction between the different large-scale IoT applications, the criteria with regard to unified data format are widely considered to be made for coordination of different systems in relevant international organizations, research institutions, and enterprises.

\section{Conflict of Interests}

The authors declare that there is no conflict of interests regarding the publication of this paper.

\section{Acknowledgments}

This work is funded by the European Framework Program (FP7) under Grant no. FP7-PEOPLE-2011-IRSES, the National Natural Science Foundation of China under Grant nos. 61073009 and 60873235 and 61103197, the National High Technology R\&D Program 863 of China under Grant no. 2011AA010101, the National Key Technology R\&D Program of China under Grant no. SQ2013GX11E00316-F03, the National Science and Technology Major Projects of China under Grant nos. SinoProbe-09-01-03 and 2012ZX01039-004-04-3, the Key Science Technology Program of Jilin Province of China under Grant no. 2011ZDGG007, the Key Scientific and Technological Project of Jilin Province of China under Grant no. 20150204035GX, and the Fundamental Research Funds for Central Universities of China under Grant no. JCKYQKJC46.

\section{References}

[1] S. Sarma, D. L. Brock, and K. Ashton, "The networked physical world-proposals for engineering the next generation of computing, commerce \& automatic identification," Auto-ID Centre White paper, 2000, http://www.foxner.com.

[2] International Telecommunication Union, ITU Internet Reports 2005: The Internet of Things, International Telecommunication Union, 2005.

[3] J. Li, Y. Zhang, D. Zhou, H. Zhang, and H. Xu, "Design and application of a new IOT reader," in Proceedings of the 2nd International Conference on Information Science and Engineering (ICISE '10), pp. 1944-1947, IEEE, Hangzhou, China, December 2010.

[4] D. Yan-E, "Design of intelligent agriculture management information system based on IoT", in Proceedings of the 4th International Conference on Intelligent Computation Technology and Automation (ICICTA '11), pp. 1045-1049, March 2011.

[5] X. Wang, W. Li, Y. Zhong, and W. Zhao, "Research on cloud logistics-based one-stop service platform for logistics center," in Proceedings of the IEEE 16th International Conference on Computer Supported Cooperative Work in Design (CSCWD '12), pp. 558-563, Wuhan, China, May 2012.

[6] L. Foschini, T. Taleb, A. Corradi, and D. Bottazzi, "M2M-based metropolitan platform for IMS-enabled road traffic management in IoT,' IEEE Communications Magazine, vol. 49, no. 11, pp. 50-57, 2011.

[7] H. Dahai, Z. Jie, Z. Yongjun, and G. Wanyi, "Convergence of sensor networks/internet of things and power grid information network at aggregation layer," in International Conference on
Power System Technology (POWERCON '10), pp. 1-6, Hangzhou, China, 2010.

[8] H. Wang, T. Zhang, Y. Quan, and R. Dong, "Research on the framework of the environmental internet of things," International Journal of Sustainable Development and World Ecology, vol. 20, no. 3, pp. 199-204, 2013.

[9] C. Du and S. Zhu, "Research on urban public safety emergency management early warning system based on technologies for the Internet of things," Procedia Engineering, vol. 45, pp. 748754, 2012.

[10] L. Dongxin and L. Tao, "The application of IOT in medical system," in Proceedings of the IEEE International Symposium on IT in Medicine and Education (ITME '11), pp. 272-275, December 2011.

[11] A. J. Jara, M. A. Zamora, and A. F. G. Skarmeta, "An architecture based on internet of things to support mobility and security in medical environments," in Proceedings of the 7th IEEE Consumer Communications and Networking Conference (CCNC '10), pp. 1-5, IEEE, Las Vegas, Nev, USA, January 2010.

[12] M. Darianian and M. P. Michael, "Smart home mobile RFIDbased internet-of-things systems and services," in Proceedings of the International Conference on Advanced Computer Theory and Engineering (ICACTE '08), pp. 116-120, December 2008.

[13] L. Atzori, A. Iera, and G. Morabito, "The internet of things: a survey," Computer Networks, vol. 54, no. 15, pp. 2787-2805, 2010.

[14] L. Xu, W. He, and S. Li, "Internet of things in industries: a survey," IEEE Transactions on Industrial Informatics, vol. 10, no. 4, pp. 2233-2243, 2014.

[15] K. Römer, O. Kasten, and F. Mattern, "Middleware challenges for wireless sensor networks," ACM SIGMOBILE Mobile Computing and Communications Review, vol. 6, no. 4, pp. 59-61, 2002.

[16] M.-M. Wang, J.-N. Cao, J. Li, and S. K. Dasi, "Middleware for wireless sensor networks: a survey," Journal of Computer Science and Technology, vol. 23, no. 3, pp. 305-326, 2008.

[17] S. M. Sadjadi and P. K. McKinley, "A survey of adaptive middleware," Report MSU-CSE-03-35, Michigan State University, 2003.

[18] K. E. Kjær, "A survey of context-aware middleware," in Proceedings of the IASTED International Conference on Software Engineering (SE '07), pp. 148-155, February 2007.

[19] M. Miraoui, C. Tadj, and C. B. Amar, "Architectural survey of context-aware systems in pervasive computing environment," Ubiquitous Computing and Communication Journal, vol. 3, no. 3, pp. 1-9, 2008.

[20] S. Bandyopadhyay, M. Sengupta, S. Maiti, and S. Dutta, "A survey of middleware for internet of things," in Recent Trends in Wireless and Mobile Networks, pp. 288-296, Springer, Berlin, Germany, 2011.

[21] C. Perera, A. Zaslavsky, P. Christen, and D. Georgakopoulos, "Context aware computing for the internet of things: a survey," IEEE Communications Surveys \& Tutorials, vol. 16, no. 1, pp. 414-454, 2014

[22] M. H. Alizai, H. Wirtz, B. Kirchen, and K. Wehrle, "Portable wireless-networking protocol evaluation," Journal of Network and Computer Applications, vol. 36, no. 4, pp. 1230-1242, 2013.

[23] M. Kovatsch, M. Lanter, and S. Duquennoy, "Actinium: a RESTful runtime container for scriptable internet of things applications," in Proceedings of the 3rd International Conference on the Internet of Things (IOT 2'12), pp. 135-142, October 2012. 
[24] C. Perera, A. Zaslavsky, P. Christen, and D. Georgakopoulos, "Sensing as a service model for smart cities supported by internet of things," Transactions on Emerging Telecommunications Technologies, vol. 25, no. 1, pp. 81-93, 2014.

[25] D. Schall, F. Skopik, and S. Dustdar, "Expert discovery and interactions in mixed service-oriented systems," IEEE Transactions on Services Computing, vol. 5, no. 2, pp. 233-245, 2012.

[26] J. Zhou, L. Hu, J. Chu, H. Lu, F. Wang, and K. Zhao, "Feature selection from incomplete multi-Sensor information system based on positive approximation in rough set theory," Sensor Letters, vol. 11, no. 5, pp. 974-981, 2013.

[27] J. Zhou, L. Hu, F. Wang, H. Lu, and K. Zhao, "An efficient multidimensional fusion algorithm for iot data based on partitioning," Tsinghua Science and Technology, vol. 18, no. 4, Article ID 6574675, pp. 369-378, 2013. 

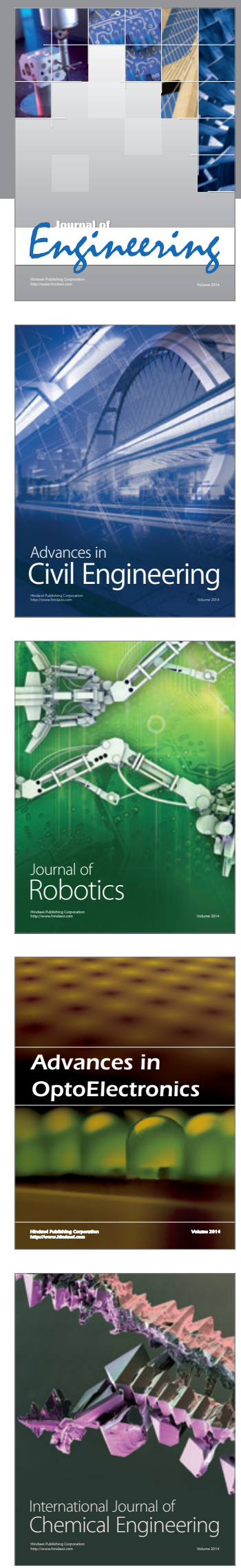

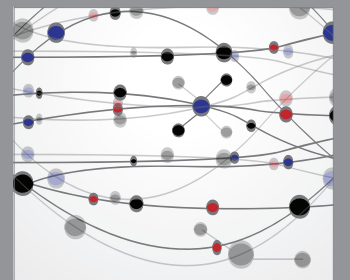

The Scientific World Journal
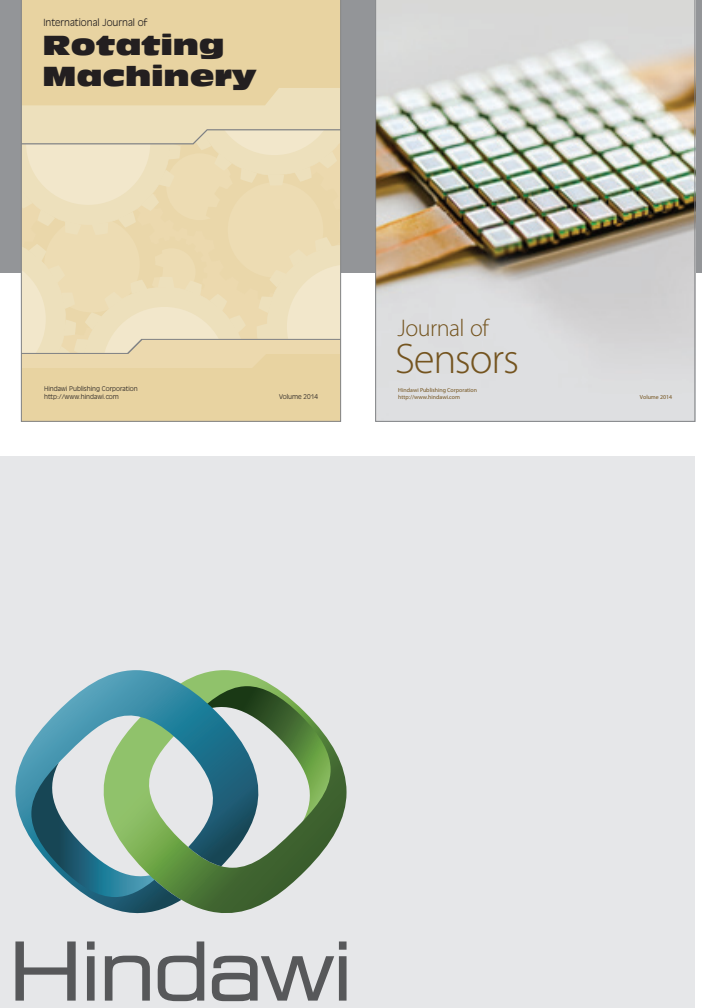

Submit your manuscripts at http://www.hindawi.com
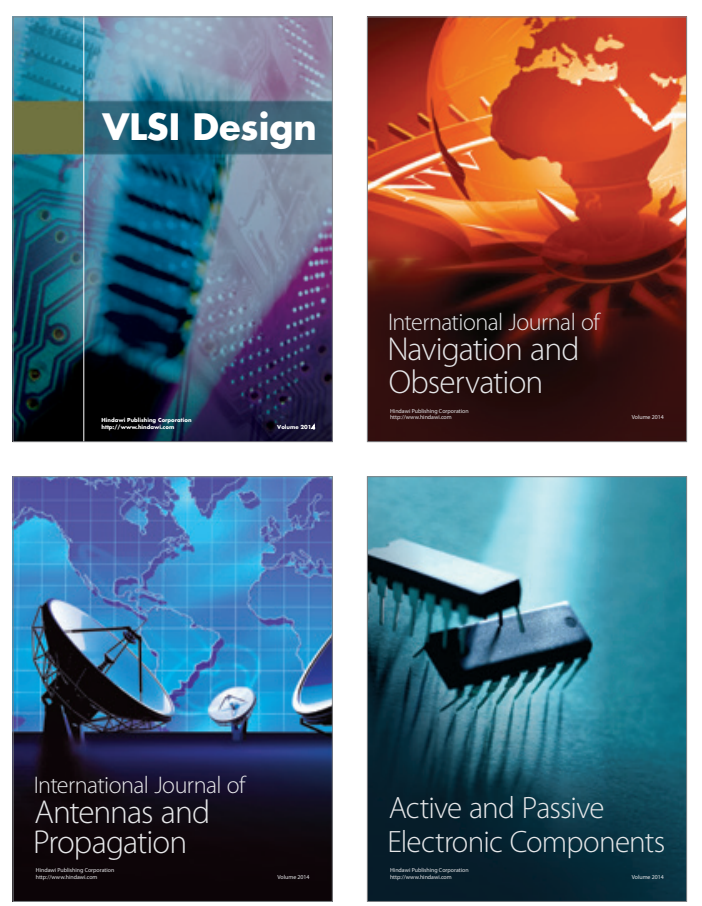
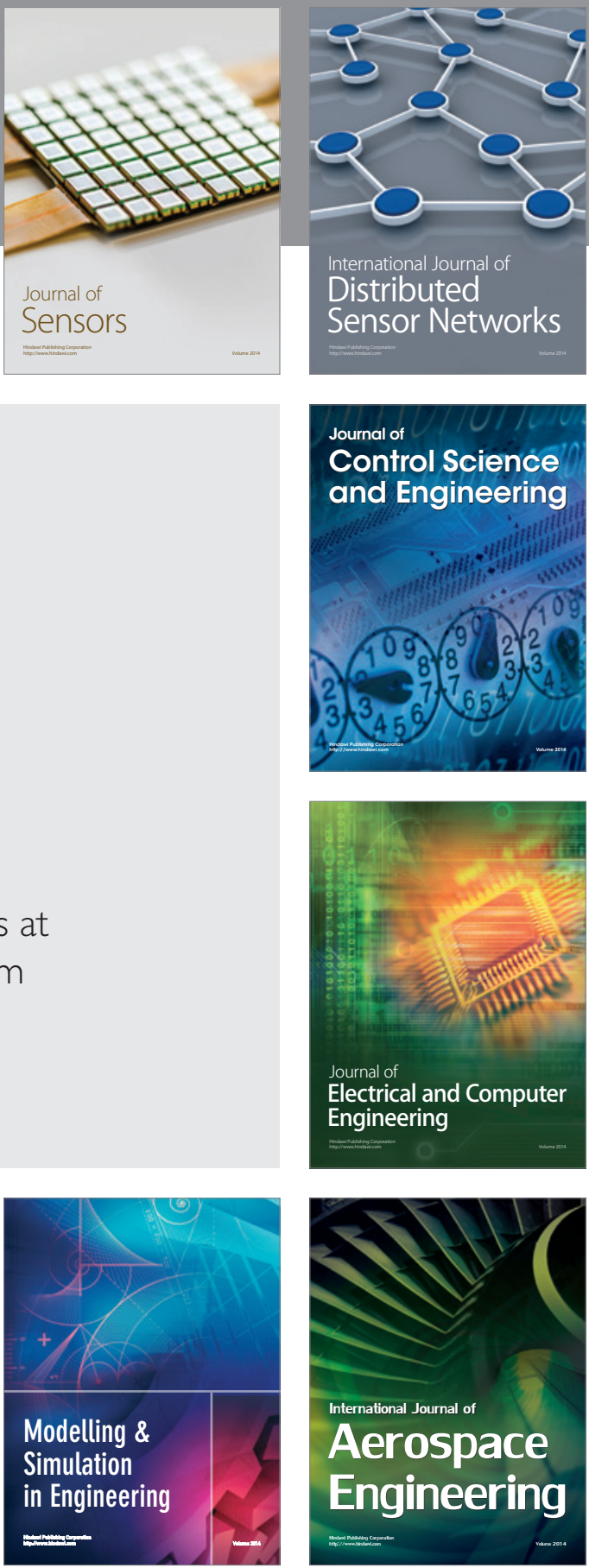

Journal of

Control Science

and Engineering
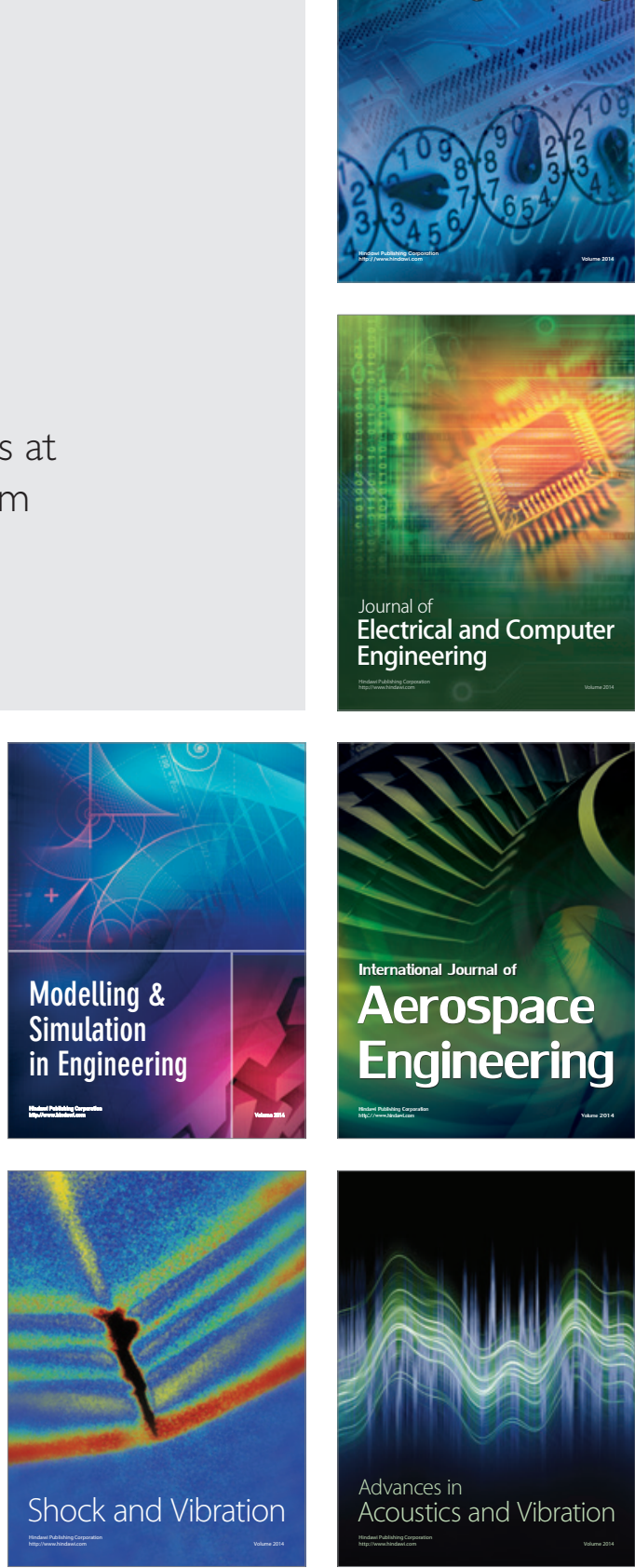